\title{
From Nanofibrous Hollow Microspheres to Nanofibrous Hollow Discs and Nanofibrous Shells
}

\author{
Zhanpeng Zhang, Peter X. Ma*
}

Nano- and microsized structures are of central importance to advanced materials and nanotechnologies, which have tremendously impacted both biomedical and physical sciences. Herein, novel emulsification and thermally induced phase separation (TIPS) techniques to fabricate linear polymers into nanofibrous hollow objects are reported for the first time. Through manipulating the emulsification conditions, the evolution of the emulsion structure can be controlled and nanofibrous hollow microspheres with a controllable opening size and nanofibrous shells can be fabricated. Through adjusting the rheological properties of the emulsions, nanofibrous hollow discs are also created. A new mechanistic hypotheses of the nanofibrous hollow object formation is proposed: the nano- and microscaled structures are independently determined by TIPS and the emulsification process, respectively. Guided by this theory, the nanofiber formation conditions for two further additional polymers (polyacrylonitrile and Nylon) under TIPS are identified, and solid/nanofibrous non-hollow/hollow microspheres are created from these two additional polymers under TIPS and emulsification for the first time. Therefore, the developed strategy is applicable to various polymer systems, and can broadly impact nano- and microfabrication technologies.

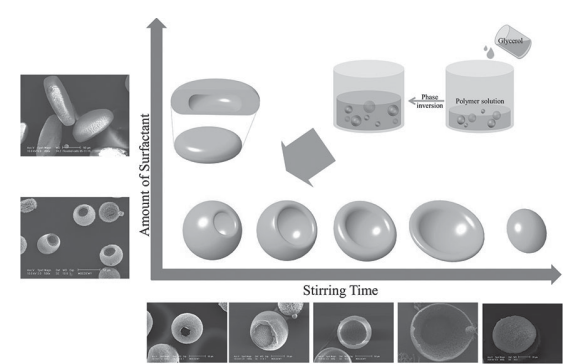

\section{Introduction}

Micro- and Nanosized spheres and hollow spheres are utilized in a wide variety of applications, ${ }^{[1-4]}$ especially for protecting delicate chemical and biological molecules as well as for controlling their release in the biomedical field. ${ }^{[5-9]}$ Recently, our laboratory developed injectable

Dr. Z. Zhang, Prof. P. X. Ma

Department of Biomedical Engineering

University of Michigan

Ann Arbor, MI 48109-1078, USA

E-mail:mapx@umich.edu

Prof. P. X. Ma

Department of Biologic and Materials Sciences

University of Michigan

Ann Arbor, MI 48109-1078, USA polymeric nanofibrous hollow microspheres and demonstrated their advantages over traditional cell carriers for knee cartilage regeneration in rabbits. ${ }^{[0]}$ However, the scientific mechanisms of the formation of the novel nanofibrous hollow microspheres were poorly understood and they could only be generated from a specifically synthesized star-shaped poly(L-lactic acid) (SS-PLLA), but not

Prof. P. X. Ma

Macromolecular Science and Engineering Center

University of Michigan

Ann Arbor, MI 48109-1078, USA

Prof. P. X. Ma

Department of Materials Science and Engineering

University of Michigan

Ann Arbor, MI 48109-1078, USA 
from linear polymers. ${ }^{[10]}$ Therefore, the star-shaped molecular structure was suspected to play an important role in the formation of nanofibrous hollow microspheres. Here we propose a new theory of nanofibrous hollow structure formation. Specifically, we hypothesize: (1) increasing the affinity of a polymer solution (including linear polymers such as a linear PLLA) to the emulsion medium initiates and stabilizes double-emulsion formation leading to hollow object formation; (2) the double-emulsion process does not interfere with a phase-separation/self-assembly process that results in nanofiber formation. ${ }^{[11,12]}$ The following experiments were carried out to test the new hypotheses and to develop new techniques to assemble linear polymers not only into nanofibrous hollow microspheres, but also into nanofibrous hollow discs and nanofibrous shells.

\section{Results and Discussions}

In one approach, a mixed solvent system (water/tetrahydrofuran (THF) mixture instead of THF alone) was chosen to dissolve a linear PLLA to form a PLLA solution with a higher affinity to glycerol (used as an emulsion medium here) than THF alone for the fabrication of nanofibrous hollow microspheres. A large amount of glycerol (with a glycerol volume at least three times larger than that of the polymer solution) was gradually added into the rigorously stirred polymer solution. Initially, glycerol was the dispersed phase in the polymer solution. Passing the phaseinversion point, while there was glycerol encapsulated inside the polymer solution spheres, the excessive glycerol became the new continuous phase. A "water-in-oil-inwater" (W/O/W) type double emulsion (glycerol-in-PLLA/ $\mathrm{THF} / \mathrm{H}_{2} \mathrm{O}$-in-glycerol) was generated. Upon quenching the double emulsion in liquid nitrogen and the subsequent extraction of the solvent and glycerol, nanofibrous hollow microspheres were obtained (Figure 1A). As demonstrated in our previous publication, ${ }^{[10]}$ using the linear PLLA in a single solvent (THF alone), nanofibrous microspheres without a hollow core were obtained (Figure 1Ba,d). When the $\mathrm{H}_{2} \mathrm{O} /$ THF ratio was $1: 20$, certain portion of the nanofibrous microspheres had a hollow core (Figure $1 \mathrm{Bb}$ ). When the $\mathrm{H}_{2} \mathrm{O} / \mathrm{THF}$ ratio was increased to $1: 10$, essentially all the formed microspheres were nanofibrous hollow microspheres (Figure 1Bc,e). All the above-formed microspheres (hollow or not) were nanofibrous (Figure 1Bf), indicating that the double-emulsion process (to form microspheres and their hollow cores) and the phase-separation/selfassembly (to form the nanofibers) processes do not interfere with one another.

In the previous study, ${ }^{[10]}$ the SS-PLLA could be considered to be an internal surfactant or self-emulsifier because there were hydroxyl groups on the SS-PLLA (both unreacted hydroxyl groups on the dendrimer core and the end hydroxyl groups on the arms), increasing the affinity to glycerol. In the present study, water could effectively function as an external surfactant since it has affinity to both THF and glycerol, reducing the surface energies associated with the interfaces. By properly choosing an $\mathrm{H}_{2} \mathrm{O}$ / THF ratio (e.g., 1:10), the double emulsion could be stabilized, leading to essentially complete nanofibrous hollow microsphere formation. Too low a ratio of $\mathrm{H}_{2} \mathrm{O}$ /THF (e.g., 1:20) might not be sufficient and therefore the double emulsion was less stable, resulting in fewer nanofibrous hollow microspheres (Figure 1Bb). It should be pointed out that there is an upper limit for the amount of water that can be added into the mixed solvent system (less than $20 \%$ for a $2 \%$ PLLA solution) since water is a nonsolvent for PLLA.

In the second approach, a glycerol derivative, Diacetin, was added into the polymer solution as an emulsifier prior to the emulsification procedure. Diacetin is a mixture of glycerol 1,2-diacetate, glycerol 1,3-diacetate, and some glycerol triacetate, i.e., esters of glycerol and acetic acid. Diacetin is biocompatible, is widely used in food and pharmaceutical formulations, ${ }^{[13]}$ and is miscible with PLLA/THF solution as well as glycerol. Thus, we rationalized that Diacetin would preferentially accumulate at the two spherical interfaces formed during the phase inversion to stabilize the double emulsion. The formation of nanofibrous hollow microspheres by this method was observed by scanning electron microscopy (SEM) when $0.05 \% \mathrm{v} / \mathrm{v}(10 \mu \mathrm{L})$ of Diacetin was added (Figure 2Aa,b). The internal structure was examined using confocal laser scanning microscopy, confirming hollow core formation (Figure 2Ac). The fabricated nanofibrous hollow microspheres of a linear PLLA using these two approaches were similar in structure to those previously generated from SS-PLLA, except that there was generally one hole on the spherical shells instead of multiple holes observed on those formed from SS-PLLA. ${ }^{[10]}$ This could stem from the fact that SS-PLLA served as a self-emulsifier, with the glycerol-philic hydroxyl groups not only stabilizing the two main spherical interfaces defining the hollow microspheres but also the holes on the spherical shells. The multiple holes were largely circumvented by the use of a linear PLLA and the use of either a solvent mixture or the addition of a separate emulsifier (e.g., Diacetin). The single hole on the spherical shell of the nanofibrous hollow spheres formed from the linear PLLA was likely resulted from the glycerol neck connecting the encapsulated glycerol core and the continuous glycerol phase upon phase inversion.

Interestingly, when the emulsifier amount was increased to $0.15 \%$, most of the hollow spherical shells folded or collapsed to some degree (Figure 2Ad-f). When the amount of the emulsifier was further increased to 


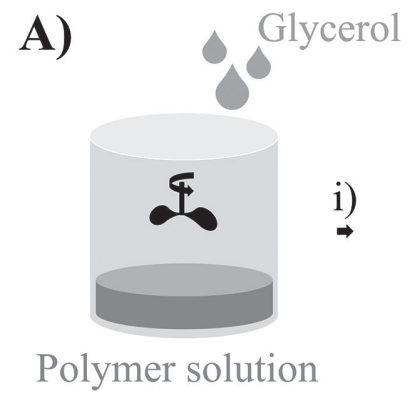

B)

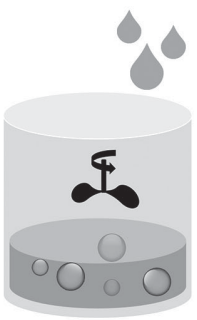

ii)

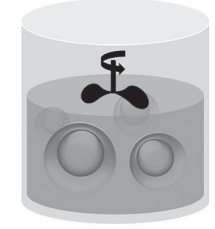

iv) iii)

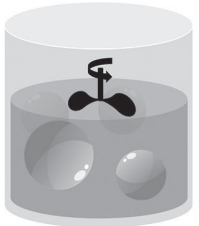

เiv)
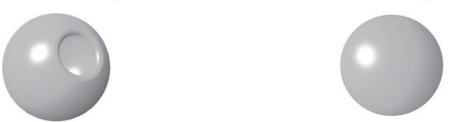

\section{$\mathrm{H}_{2} \mathrm{O}$ content}

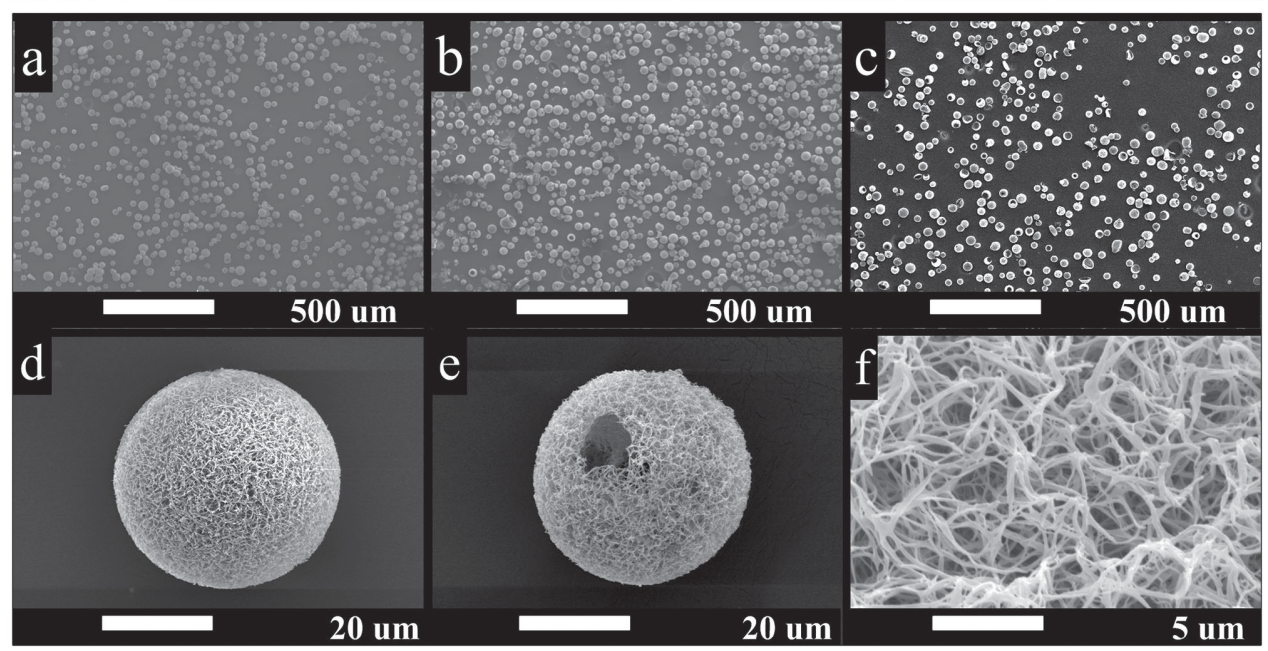

Figure 1. A) A schematic illustration of the emulsification and TIPS process for the fabrication of microspheres, including steps: i) the addition of glycerol into polymer solution under stirring, generating glycerol microdroplets; ii) phase inversion, generating glycerol-in-polymer solution-in-glycerol double emulsions; iii) possibly the transformation into polymer solution-in-glycerol single emulsions; iv) quenching in liquid nitrogen, solvent and glycerol extraction in water, and freeze-drying. B) Nanofibrous microspheres (with diameter ranging from $60-90 \mu \mathrm{m}$ ) from emulsion of a,d) $20 \mathrm{~mL} 2 \%$ PLLA/THF solution, b) PLLA/THF/ $\mathrm{H}_{2} \mathrm{O}$ solution with $\mathrm{H}_{2} \mathrm{O} / \mathrm{THF}$ ratio of 1:20, and c,e) PLLA/THF/ $\mathrm{H}_{2} \mathrm{O}$ solution with $\mathrm{H}_{2} \mathrm{O} / \mathrm{THF}$ ratio of 1:10 in $100 \mathrm{~mL}$ glycerol under stirring for $10 \mathrm{~s}$. f) All of them had a nanofibrous structure.

$0.3 \%$, the hollow microspheres transitioned into hollow discs (Figure 2Ag-i). Despite the change of the overall shape, the nanofibrous (NF) structure was maintained, once again indicating that the emulsification process and phase-separation/self-assembly process do not interfere one another. In addition to being an emulsifier, Diacetin has been used as a plasticizer to soften rigid plastics. ${ }^{[13]}$ The addition of Diacetin into the polymer solution lowers the solution viscosity. However, a high polymer solution viscosity is needed to stabilize the spherical emulsion. ${ }^{[14]}$ The intermediate level of Diacetin might lead to regional softening and the formation of weak spherical polymer shells, which tend to fold and collapse through the softened regions. The nanofibrous hollow disc formation could also be attributed to the Diacetin's softening effect. The addition of a larger amount of Diacetin likely softens the entire spherical polymer solution shells and changes the rheological properties, allowing the coordinated deformation from spheres into discs under the shear stresses of vigorous stirring. These results therefore have demonstrated that by adjusting the amount of emulsifier, we are not only able to fabricate nanofibrous hollow microspheres but also nanofibrous hollow discs using a commercial linear PLLA.

In the third approach, we also added the emulsifier Diacetin into the polymer solution to stabilize the generated double emulsion. However, instead of varying the amount of the emulsifier, we utilized the thermodynamic metastability of the double emulsion and the associated structural transition over time to obtain the desired microcarrier structures. We first demonstrated that variable size of the openings on the nanofibrous hollow

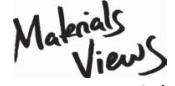

www.MaterialsViews.com 


\section{A)

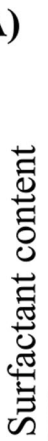

B)

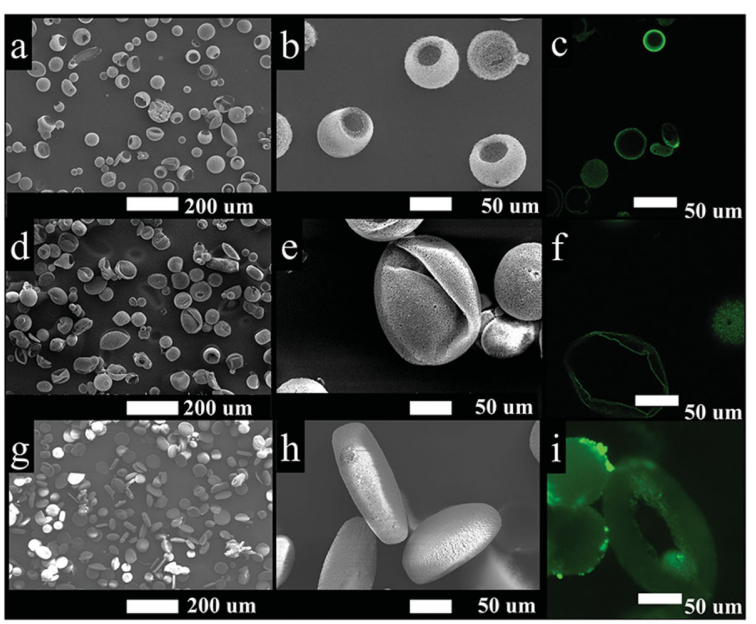

C)

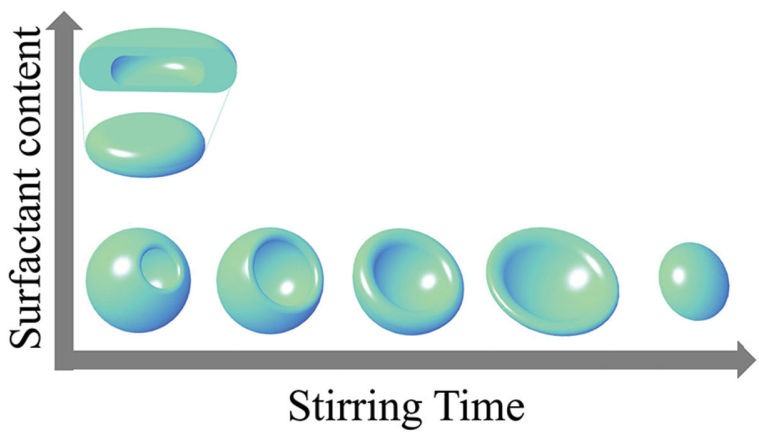

Stirring Time

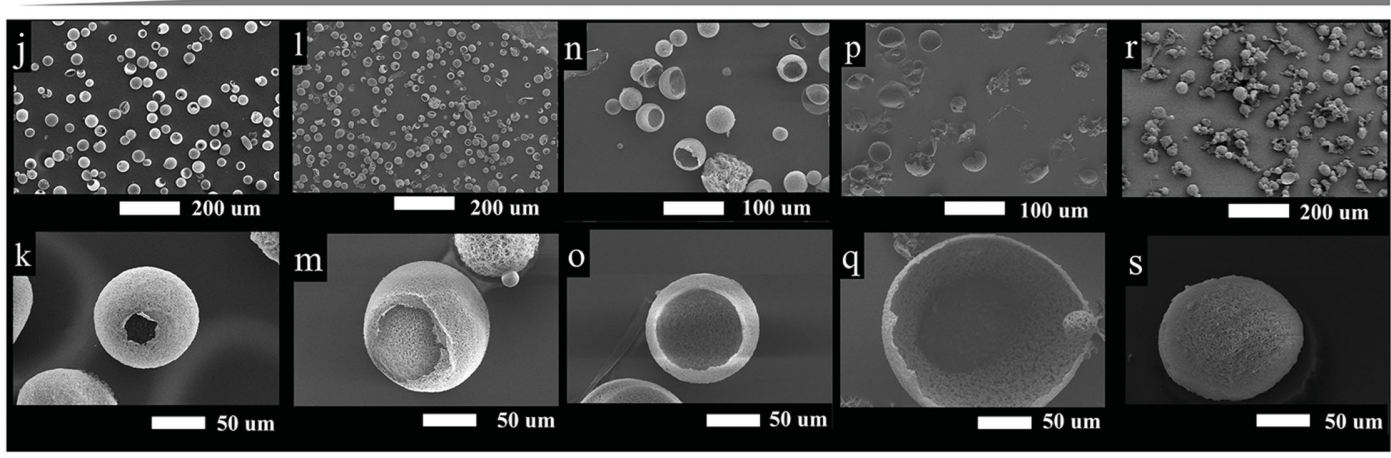

Figure 2. A) SEM images of nanofibrous microcarriers (with diameter ranging from 60 to $90 \mu \mathrm{m}$ ) prepared from an emulsion of a,b) $20 \mathrm{~mL}$ $2 \%$ PLLA/THF solution and 0.05\% (10 $\mu \mathrm{L}$ ) Diacetin; d,e) $0.15 \%(30 \mu \mathrm{L})$ Diacetin; and g,h) $0.3 \%(60 \mu \mathrm{L})$ Diacetin in $100 \mathrm{~mL}$ glycerol under stirring for 10 s. c,f,i) Their internal structure was observed under confocal microscopy after being stained with FITC-tagged BSA. B) Nanofibrous microcarriers with various structures prepared from emulsion of $20 \mathrm{~mL} 2 \%$ PLLA/THF solution with $0.05 \%$ Diacetin in $100 \mathrm{~mL}$ glycerol under stirring for: j,k) $10 \mathrm{~s} ; \mathrm{l}, \mathrm{m}) 1 \mathrm{~min} ; \mathrm{n}, \mathrm{o}) 5 \mathrm{~min} ; \mathrm{p}, \mathrm{q}) 10 \mathrm{~min}$; and r,s) 1 h. C) A schematic illustration summarizing the structural variations of nanofibrous microcarriers in response to different emulsifier content and emulsification duration.

microspheres could be achieved. Under the nanofibrous hollow microsphere-forming conditions (with $0.05 \%$ of Diacetin), we collected the microcarriers at different stirring times, from $10 \mathrm{~s}$ to $1 \mathrm{~min}, 5 \mathrm{~min}, 10 \mathrm{~min}$, and $1 \mathrm{~h}$. It was found that the size of the pore openings increased as stirring time increased (Figure $2 \mathrm{~B}$ ). When the stirring time was $10 \mathrm{~s}$, the size of pore openings on the microspheres was about $20 \mu \mathrm{m}$ on average when the average microsphere diameter was in the range of $60-90 \mu \mathrm{m}$ (Figure 2Bj,k). However, when the stirring time was $1 \mathrm{~min}$, the average size of pore openings increased to about $25 \mu \mathrm{m}$ for microspheres in the same size range (Figure 2Bl,m). When the stirring time was $5 \mathrm{~min}$, the hollow microspheres became more like bowls (nanofibrous hollow hemispheres) with a significantly larger opening size of about $63 \mu \mathrm{m}$ (Figure 2Bn,o). When the stirring time was extended to $10 \mathrm{~min}$, shallow nanofibrous shells were obtained (Figure 2Bp,q). When the stirring time was further extended to $1 \mathrm{~h}$, smaller and essentially spherical nanofibrous particles were formed, most of which do not have a hollow core (Figure $2 \mathrm{Br}, \mathrm{s}$ ).

There were two possible key factors that contributed to the structural transition from small opening size to larger opening size of the nanofibrous hollow microspheres, and later to nanofibrous shallow shells, and smaller nanofibrous nonhollow microspheres. One was likely the softening effect of Diacetin, which allowed for easier shape change. The other factor is the thermodynamic instability or metastability of the hollow microspheres. The higher system-free energy due to the existence of the two interfaces (between core and shell, and between shell and the outer medium) likely drove the reduction of the interfacial areas. However, Diacetin likely reduced the free energy difference and substantially slowed down the shape change process. With $0.05 \%$ emulsifier and vigorous stirring, the originally concentric spherical polymer solution shell and the encapsulated glycerol core might slowly move off-center away from each other to reduce 


\section{DMF: formic acid}

A)

PAN
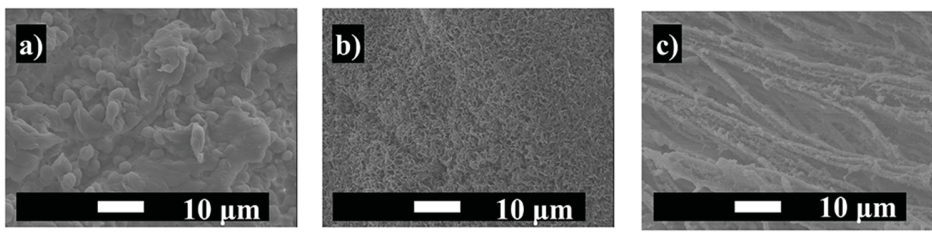

$\mathrm{H}_{2} \mathrm{O}: \mathrm{DMSO}$
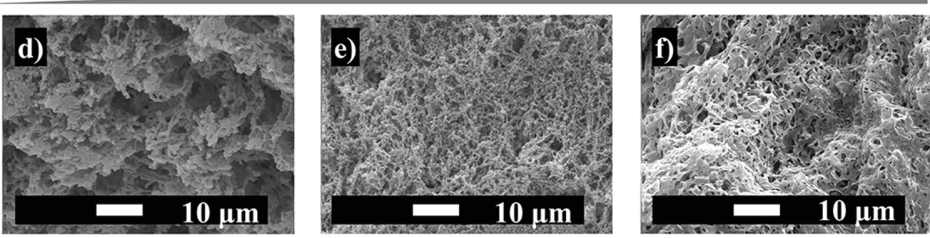

B)

NF-MS

NF-HMS

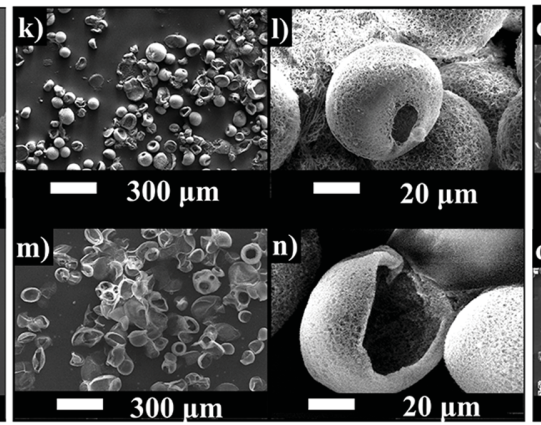

S-HMS

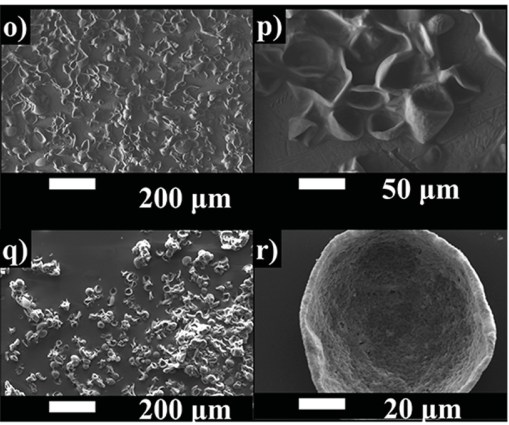

Figure 3. SEM micrographs of A) Nylon matrices fabricated by a) TIPS from $2 \%$ Nylon solution in formic acid or b) in a solvent mixture of formic acid and DMF with a mixing ratio of 3:1 or c) 1:1; PAN matrices fabricated by d) TIPS from $2 \%$ PAN solution in DMSO or e) in solvent mixture of DMSO and $\mathrm{H}_{2} \mathrm{O}$ with a mixing ratio of 9:1 or f) 7:1. B) NF-MS fabricated from g,h) Nylon and i,j) PAN via phase separation and surfactant-free emulsification techniques; NF-HMS fabricated from $k, l)$ Nylon and $m, n$ ) PAN via surfactant-assisted emulsification and phase separation techniques; S-HMS fabricated from o,p) Nylon and q,r) PAN via surfactant-assisted emulsification technique.

the interfacial areas. As they moved off-center, the size of the neck connecting the encapsulated glycerol and the surrounding glycerol increased, and therefore the size of the generated openings on the hollow microspheres increased. Once passing the hollow hemisphere stage, the polymer solution changed into shallow shells. Given a long enough time, these polymer solution domains transitioned into the more stable (lower energy) smaller nonhollow microspheres (equivalent to the escape of the initially encapsulated glycerol core and the healing of the hollow shell into a sphere). The structural changes of the second and third approaches (varying emulsifier content and stirring duration before quenching the polymer solution) are summarized in Figure $2 \mathrm{C}$. Due to the slow evolution of the emulsion structure, different microcarrier structures can be arrested in liquid nitrogen in a robust manner. However, varying the stirring time was less effective in the first approach, where $\mathrm{H}_{2} \mathrm{O} / \mathrm{THF}$ solvent mixture was used (data not shown), probably because the addition of water had little effect on the rheological properties of the polymer (water is not a solvent or effective plasticizer for PLLA).

Biomaterials' physical shape and structural feature size on the micro and nanoscales are increasingly recognized to play important roles in their function as cell carriers for tissue engineering ${ }^{[10,12,15-21]}$ and as vehicles for controlled or targeted therapeutic delivery. ${ }^{[22-31]}$ Recently, novel nanofibrous hollow polymer microspheres have been developed for the first time in our laboratory and demonstrated to be an excellent cell carrier for cartilage regeneration. ${ }^{[10]}$ However, the nanofibrous hollow microspheres could only be self-assembled from a star-shaped polymer, which was synthesized through a costly and more time-consuming process than commercially available linear polymers. In this work, we formed generalized hypotheses of nanofibrous hollow structure formation and developed techniques to fabricate nanofibrous hollow microspheres using a conventional linear PLLA for the first time. In addition, we developed techniques to control the open hole size on the nanofibrous hollow

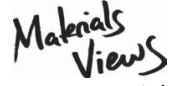

www.MaterialsViews.com
Macromol. Rapid Commun. 2015, 36, 1735-1741

(c) 2015 WILEY-VCH Verlag GmbH \& Co. KGaA, Weinheim 
microspheres. Furthermore, we developed new and facile fabrication techniques to generate nanofibrous hollow discs and nanofibrous shells for the first time. These are important advances in the fabrication of complex nano/ micromaterials. The new microcarriers will be evaluated for applications in tissue regeneration and biomolecule deliveries in future studies.

While this proposed theory has been supported by the data from linear PLLA, can other polymers be utilized to generate nanofibrous hollow micro objects? We have found that Nylon and polyacrylonitrile (PAN) can also be fabricated into nanofibrous materials via phaseseparation (Figure 3A). Using our technology developed from PLLA, we have experimentally demonstrated that both Nylon and PAN can be fabricated into nanofibrous microspheres and nanofibrous hollow microspheres (Figure $3 \mathrm{Bg}-\mathrm{n}$ ), supporting our new generalized hypotheses. When solvents that do not allow nanofibrous structure formation are chosen, we can also use our techniques to fabricate hollow micro objects that are not nanofibrous (Figure 3Bo-r), supporting our hypothesis that the phase-separation process (nanofiber formation) and the hollow object formation process do not interfere with one another. In addition, we have demonstrated that the solvent exchange process (using ice water to replace organic solvent) does not affect the nanofibrous structure (comparing Figures S1 and S2 in the Supporting Information), which should have formed during the phase-separation process before the solvent exchange process. The above supplementary data are consistent with the new hypotheses and have expanded the polymer types for nanofibrous hollow microobject fabrication.

\section{Conclusions}

In addition to biomolecule release and tissue regeneration, micro- and nanoparticles have found a wide variety of applications in electronic displays, ${ }^{[32]}$ photonic crystal preparation, ${ }^{[33-35]}$ self-healing structural materials, ${ }^{[36]}$ separation and catalysis, ${ }^{[37,38]}$ highly sensitive protein detection, ${ }^{[39,40]}$ and so on. The new theory may be utilized to generate nanofibrous hollow spheres/discs/shells from additional polymers and materials, which may potentially be employed in biocatalysis, separation, cosmetics, food additives, and other applications.

\section{Experimental Section}

Materials: PLLA with an inherent viscosity of approximately 1.6 was purchased from Boehringer Ingelheim (Ingelheim, Germany). Tetrahydrofuran (THF), glycerol, Diacetin (a mixture of glycerol 1, 2-diacetate, glycerol 1,3-diacetate, and some glycerol triacetate) were purchased from Aldrich Chemical (Milwaukee, WI). Deionized water was obtained with a Milli-O water filter system from Millipore Corporation (Bedford, MA). Polyacrylonitrile (PAN) (with an average molecular weight of $150 \mathrm{kDa}$ ) and Nylon6/6 were purchased from Aldrich Chemical (Milwaukee, WI). Formic acid, dimethylformamide (DMF), dimethyl sulfoxide (DMSO), silicon oil, and olive oil were purchased from Aldrich Chemical (Milwaukee, WI) and used as received. Poly(ethylene glycol)-block-poly(propylene glycol)block-poly(ethylene glycol) (PEO-PPO-PEO) with $\mathrm{Mn}$ of $5800 \mathrm{Da}$ and viscosity of $350000 \mathrm{cps}\left(60^{\circ} \mathrm{C}\right)$ was purchased from Aldrich Chemical (Milwaukee, WI) and used as received.

Surfactant-Free Emulsification Using Solvent Mixture: In the first approach, a mixture of THF and $\mathrm{H}_{2} \mathrm{O}$ with different mixing ratios $\left(\mathrm{H}_{2} \mathrm{O} / \mathrm{THF}=1: 20\right.$ and $\left.1: 10, \mathrm{v} / \mathrm{v}\right)$ was used as the solvent to prepare the PLLA solutions at $50{ }^{\circ} \mathrm{C}$ with a concentration of $2.0 \%$ $(\mathrm{w} / \mathrm{v})$. Under rigorous mechanical stirring (speed 7, MAXIMA, Fisher Scientific Inc.), glycerol $\left(50^{\circ} \mathrm{C}\right)$ with a volume at least three times larger than that of PLLA solution was gradually added into the polymer solution, and the stirring continued for another $5 \mathrm{~min}$. The mixture was then quickly poured into liquid nitrogen. After $10 \mathrm{~min}$, ice/water mixture was added for solvent exchange for $24 \mathrm{~h}$. The microspheres were then sieved and washed with distilled water for a few times to remove residual glycerol. The microspheres were then lyophilized for $2 \mathrm{~d}$.

Surfactant-Assisted Emulsification: In the second and third approaches, $0.4 \mathrm{~g}$ PLLA was dissolved in $20 \mathrm{~mL}$ THF at $50{ }^{\circ} \mathrm{C}$ to achieve a concentration of $2.0 \%(\mathrm{w} / \mathrm{v})$. Different amounts of Diacetin $(0.05 \%, 0.15 \%$, or $0.3 \%(\mathrm{v} / \mathrm{v}))$ were then added into the polymer solution. Under rigorous mechanical stirring (speed 7, MAXIMA, Fisher Scientific Inc.), glycerol $\left(50{ }^{\circ} \mathrm{C}\right.$ ) with a volume at least three times larger than that of the PLLA solution was gradually added into the polymer solution, and the stirring continued for another $10 \mathrm{~s}, 1 \mathrm{~min}, 5 \mathrm{~min}, 10 \mathrm{~min}$, or longer. The mixture was then quickly poured into liquid nitrogen. After 10 $\mathrm{min}$, ice/water mixture was added for solvent exchange for $24 \mathrm{~h}$. The microspheres were then sieved and washed with distilled water for a few times to remove residual Diacetin and glycerol. The microspheres were then lyophilized for $2 \mathrm{~d}$. It should be noted that the emulsification conditions described above typically generate microcarriers with a size ranging from $10 \mu \mathrm{m}$ to $500 \mu \mathrm{m}$ in diameter; microcarriers with a smaller size can be obtained by changing the emulsification conditions, such as increasing the amount of the surfactant or stirring speed.

Preparation of Nylon and Polyacrylonitrile (PAN) Nanofibers: Nylon6/6 was dissolved in pure formic acid and formic acid/ dimethylformamide (DMF) solvent mixture with a mixing ratio of $3: 1$ or $1: 1(\mathrm{v} / \mathrm{v})$ to make a $2 \%(\mathrm{w} / \mathrm{v})$ polymer solution at $80{ }^{\circ} \mathrm{C}$. PAN was dissolved in pure DMSO and DMSO $/ \mathrm{H}_{2} \mathrm{O}$ solvent mixture with a mixing ratio of 9:1 or 7:1 (v/v) to make a $2 \%(\mathrm{w} / \mathrm{v})$ polymer solution at $80{ }^{\circ} \mathrm{C}$. The polymer solutions were quickly transferred into liquid nitrogen to induce phase separation. After $10 \mathrm{~min}$, the frozen polymer gels were immersed into ice water for solvent exchange for $4 \mathrm{~h}$. The polymer foams were subsequently frozen at $-80^{\circ} \mathrm{C}$ for $2 \mathrm{~h}$ and freeze-dried overnight.

Preparation of Nylon and PAN Microspheres with Different Nano and Micro Features: Nylon NF-MS were fabricated under the same process that PLLA NF-MS were fabricated, except that Nylon6/6 was dissolved in a mixture of formic acid and 
DMF (formic acid:DMF = 3:1, v/v) to form a $2.0 \%(\mathrm{w} / \mathrm{v}$ ) polymer solution for emulsification in olive oil $\left(50{ }^{\circ} \mathrm{C}\right)$. After quenching in liquid nitrogen and solvent extraction in ice water, the microspheres were then sieved and washed with hexane once, ethanol once, and distilled water for four times to remove the oil. Nylon NF-HMS were fabricated the same way Nylon NF-MS were made, except that $0.2 \%(\mathrm{w} / \mathrm{v})$ surfactant poly(ethylene glycol)-block-poly(propylene glycol)-block-poly(ethylene glycol) (PEO-PPO-PEO) was added into the Nylon polymer solution prior to the emulsification and phase separation steps. Nylon S-HMS were fabricated the same way as Nylon NF-HMS, except that the solvent to dissolve Nylon was formic acid alone ( $2 \% \mathrm{w} / \mathrm{v}$ Nylon in formic acid).

PAN NF-MS were fabricated under the same process that Nylon NF-MS were made, except that PAN was dissolved in a mixture of DMSO and deionized water (DMSO: $\mathrm{H}_{2} \mathrm{O}=9: 1, \mathrm{v} / \mathrm{v}$ ) for emulsification in silicon oil $\left(50^{\circ} \mathrm{C}\right)$. PAN NF-HMS were fabricated under the same process that PAN NF-MS were made, except that $0.2 \%(\mathrm{w} / \mathrm{v})$ PEO-PPO-PEO were added into the PAN polymer solution before the emulsification and phase separation. PAN S-HMS were created by emulsifying $2 \%(w / v)$ PAN in pure DMSO under the same emulsification process that PAN NF-HMS were fabricated.

Observation: The morphologies of the microspheres, discs, or shells were examined using SEM (Philips XL30 FEG) with an accelerating voltage of $10 \mathrm{kV}$. The samples were coated with gold for $120 \mathrm{~s}$ using a sputter coater (DeskII, Denton Vacuum Inc.).

The internal structure of the spheres/discs was examined using confocal laser scanning microscopy (CLSM) (Nikon Eclipse C1). Fluorescein isothiocyanate (FITC)-conjugated bovine serum albumin (BSA) was adsorbed on the nanofibrous microcarriers to visualize their structures under confocal microscopy.

\section{Supporting Information}

Supporting Information is available from the Wiley Online Library or from the author.

Acknowledgements: The authors gratefully acknowledge the research grant support from the NSF (DMR-1206575), NIH (NIDCR DE015384, DE017689, and DE022327), and DOD (W81XWH-12-2-0008).

Received: June 14, 2015; Revised: July 14, 2015; Published online: August 6, 2015; DOI: 10.1002/marc.201500342

Keywords: microstructures; nanofibers; phase separation; polymers; self-assembly

[1] H. Deng, X. Li, O. Peng, X. Wang, J. Chen, Y. Li, Angew. Chem.Int. Ed. 2005, 44, 2782.

[2] Y. Deng, D. Oi, C. Deng, X. Zhang, D. Zhao, J. Am. Chem. Soc. 2008, 130, 28.

[3] B. Jeong, Y. H. Bae, D. S. Lee, S. W. Kim, Nature 1997, 388, 860.

[4] J. S. Oh, L. N. Dang, S. W. Yoon, P. C. Lee, D. O. Kim, K. J. Kim, J. D. Nam, Macromol. Rapid Commun. 2013, 34, 504.
[5] R. Langer, Nature 1998, 392, 5

[6] R. V. Parthasarathy, C. R. Martin, Nature 1994, 369, 298.

[7] X. C. Xiao, L. Y. Chu, W. M. Chen, S. Wang, Y. Li, Adv. Funct. Mater. 2003, 13, 847.

[8] J. Guo, W. Yang, C. Wang, Adv. Mater. 2013, 25, 5196.

[9] D. L. Elbert, Acta Biomater. 2011, 7, 31.

[10] X. Liu, X. Jin, P. X. Ma, Nat. Mater. 2011, 10, 398

[11] P. X. Ma, R. Zhang, J. Biomed. Mater. Res. 1999, 46, 60.

[12] P. X. Ma, Adv. Drug Delivery Rev. 2008, 60, 184.

[13] D. Lourdin, L. Coignard, H. Bizot, P. Colonna, Polymer 1997, 38, 5401.

[14] N. Garti, A. Aserin, Adv. Colloid Interface Sci. 1996, 65, 37.

[15] S. Mitragotri, J. Lahann, Nat. Mater. 2009, 8, 15.

[16] A. P. McGuigan, B. Leung, M. V. Sefton, Nat. Protocols 2006, 1, 2963.

[17] A. Khademhosseini, R. Langer, J. Borenstein, J. P. Vacanti, Proc. Natl. Acad. Sci. USA 2006, 103, 2480.

[18] W. J. Chung, J. W. Oh, K. Kwak, B. Y. Lee, J. Meyer, E. Wang, A. Hexemer, S. W. Lee, Nat. 2011, 478, 364.

[19] Y. T. Matsunaga, Y. Morimoto, S. Takeuchi, Adv. Mater. 2011, 23, H90.

[20] H. Tan, O. Shen, X. Jia, Z. Yuan, D. Xiong, Macromol. Rapid Commun. 2012, 33, 2015.

[21] Z. Zhang, R. L. Marson, Z. Ge, S. C. Glotzer, P. X. Ma, Adv. Mater. 2015, 27, 3947.

[22] H. Cabral, Y. Matsumoto, K. Mizuno, O. Chen, M. Murakami, M. Kimura, Y. Terada, M. R. Kano, K. Miyazono, M. Uesaka, N. Nishiyama, K. Kataoka, Nat. Nanotechnol. 2011, 6, 815.

[23] D. Peer, J. M. Karp, S. Hong, O. C. Farokhzad, R. Margalit, R. Langer, Nat. Nanotechnol. 2007, 2, 751.

[24] C. Wang, O. Ge, D. Ting, D. Nguyen, H. R. Shen, J. Chen, H. N. Eisen, J. Heller, R. Langer, D. Putnam, Nat. Mater. 2004, 3, 190.

[25] J. B. Lee, Y. H. Roh, S. H. Um, H. Funabashi, W. Cheng, J. J. Cha, P. Kiatwuthinon, D. A. Muller, D. Luo, Nat. Nanotechnol. 2009, 4, 430.

[26] K. A. Woodrow, Y. Cu, C. J. Booth, J. K. Saucier-Sawyer, M. J. Wood, W. M. Saltzman, Nat. Mater. 2009, 8, 526.

[27] A. S. Utada, E. Lorenceau, D. R. Link, P. D. Kaplan, H. A. Stone, D. A. Weitz, Science 2005, 308, 537.

[28] S. H. Im, U. Y. Jeong, Y. N. Xia, Nat. Mater. 2005, 4, 671.

[29] Z. Zhang, M. J. Gupte, X. Jin, P. X. Ma, Adv. Funct. Mater. 2015, 25,350 .

[30] X. Kang, Z. Cheng, D. Yang, P. Ma, M. Shang, C. Peng, Y. Dai, J. Lin, Adv. Funct. Mater. 2012, 22, 1470.

[31] S. Hwang, J. Lahann, Macromol. Rapid Commun. 2012, 33, 1178.

[32] B. Comiskey, J. D. Albert, H. Yoshizawa, J. Jacobson, Nature 1998, 394, 253.

[33] J. E. G. J. Wijnhoven, W. L. Vos, Science 1998, 281, 802.

[34] S. W. Choi, I. W. Cheong, J. H. Kim, Y. Xia, Small 2009, 5, 454.

[35] A. Xia, J. Hu, C. Wang, D. Jiang, Small 2007, 3, 1811.

[36] S. R. White, N. R. Sottos, P. H. Geubelle, J. S. Moore, M. R. Kessler, S. R. Sriram, E. N. Brown, S. Viswanathan, Nature 2001, 409, 794.

[37] F. Svec, J. M. J. Frechet, Science 1996, 273, 205.

[38] S. Agrawal, A. Kumar, M. J. Frederick, G. Ramanath, Small 2005, 1, 823.

[39] J. M. Nam, C. S. Thaxton, C. A. Mirkin, Science 2003, 301, 1884.

[40] R. Zhang, H. Chen, C. Wu, X. Wang, Z. Gu, Macromol. Rapid Commun. 2005, 26, 1469.

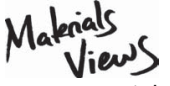

www.MaterialsViews.com
Macromol. Rapid Commun. 2015, 36, 1735-1741

(c) 2015 WILEY-VCH Verlag GmbH \& Co. KGaA, Weinheim 